\title{
Traveling in Time: A Time-Left Analogue for Humans
}

\author{
J. H. Wearden \\ Manchester University
}

\begin{abstract}
Two experiments studied normal humans in an analogue of the time-left procedure of J. Gibbon and R. M. Church (1981). In Experiment 1 the "standard" alternative $(S)$ was always half the length of the "comparison" time-left link $(C)$, and $S$ ranged from 4 to 8 s. Humans showed an increasing preference for the time-left alternative with increasing elapsed time in the interval, and indifference points strongly supported the idea of a linear, rather than a logarithmic, time scale. Experiment 2 used some conditions in which $S$ was greater or less than $C / 2$, and preference for the time-left alternative varied systematically with the $S / C$ ratio. Data from both experiments showed reasonable superposition, suggesting underlying scalar timing processes in time left in humans.
\end{abstract}

One of the many legacies of the late John Gibbon to the psychology of time is a fascinating, if somewhat complex, procedure for studying animal timing, time left (Gibbon \& Church, 1981). The time-left procedure is of particular interest because it addresses a perplexing problem in time psychology, that of how internal, subjective time and external, clock-measured time are related: in the words of Gibbon (1986) himself, "how time flies" (p. 105). It is a commonplace of psychophysics that the growth of sensation is not usually a linear function of the physical magnitude of the stimulus used, with various sorts of power functions usually describing data better than linear relations (Stevens, 1957; see Marks, 1974, for a discussion of the history of psychophysical scaling). According to scalar expectancy theory (SET; Gibbon, Church, \& Meck, 1984), on the other hand, the growth of subjective time as real time passes is a simple linear function, one in which subjective time reflects real time with almost perfect accuracy on average. Among alternatives proposed to such a linear relation between subjective and real time have been power function relations (Eisler, 1975, 1976) and the recent proposition of Staddon and Higa (1999) that subjective and objective time are logarithmically related.

Attempts to distinguish between linear and logarithmic time scales are bedeviled by the obvious fact that the internal time scale an organism possesses can be revealed only in its behavior, and in many cases the fact that behavior varies as a nonlinear function of real time can be reconciled with an underlying linear subjective time scale. This reconciliation is made easier by modern accounts of timing such as SET, which are multiprocess theories, involving memory and decision mechanisms as well as timing mechanisms; thus, some choice of memory or decision processes (or the addition of some other factors) may easily reconcile the data and theory (Wearden, 1999). A complete review of how this is done would be inappropriate here, but two examples are illustrative.

I am grateful to Clare Cave, Clare McFarlane, and Alastair Walmsley for running the participants in the two experiments, and also to the late John Gibbon for his interest and enthusiasm during the lengthy, and often discouraging, search for an appropriate time-left analogue for humans.

Correspondence concerning this article should be addressed to J. H. Wearden, Department of Psychology, Manchester University, Manchester M13 9PL, United Kingdom. E-mail: wearden@psy.man.ac.uk
Studies of the behavior of rats and pigeons on reinforcement schedules involving temporal constraints or temporal requirements have produced many examples of nonlinear relations between behavioral measures and clock time. For example, Lowe, Harzem, and Spencer (1979), in a careful study of rats and pigeons on fixed-interval (FI) schedules of reinforcement, found that the mean postreinforcement pause (the time from food delivery to the first response in the interval) varied as a power function of the FI value, with an exponent such that the pause was an increasingly small fraction of the interval length as the FI value increased. (Platt, 1979, discusses similar power-function relations between behavioral measures and imposed temporal constraints from other reinforcement schedules.) If we assume that the postreinforcement pause is a measure of the animal's "estimate" of when food is due, the Lowe et al. results clearly violate linear timing, but Wearden (1985) reconciled power functions and linear timing in a simple manner. Suppose that there are two sources of control over the response that terminates the pause. One is a linear-timing process (i.e., the response is timed to occur on average at some fraction of the FI value, as this is varied), but the other is a nontiming process of response initiation, a tendency to respond without regard for elapsed time in the interval, which has some small constant probability of producing a response each second. Obviously, as the FI value increases, it becomes more and more likely that the pause will be terminated by the nontiming process, and computer simulation showed that these two processes acting together produced a power-function relation between mean postreinforcement pause and FI value.

Another example comes from bisection tasks. In the usual procedure used with rats (Church \& Deluty, 1977), animals are initially trained to make a response on one lever after a short standard duration (e.g., a light lasting 2 s) and a response on another lever after a long standard duration (e.g., $8 \mathrm{~s}$ ). When the discrimination has been mastered to a high degree of accuracy, a range of durations is presented (the short and long standards, as well as durations in between them), and the response to each is noted during unreinforced testing. The usual way of displaying behavior is to plot a psychophysical function in the form of the proportion of "long" responses (i.e., response that would have been reinforced after the long standard duration) plotted against stimulus duration. One measure of interest derived from this psycho- 
physical function is the bisection point (the duration that gives rise to $50 \%$ long responses), and studies with nonhumans almost universally find this at the geometric mean of the short and long standards (the square root of their product, $4 \mathrm{~s}$ in the example given above), rather than at the arithmetic mean (5 $\mathrm{s}$ in the example given). If the bisection point is some kind of "perceptual midpoint" of the short and long standards, then geometric mean bisection appears to support logarithmic time scaling (as Church \& Deluty, 1977, originally suggested). However, Gibbon $(1981,1986)$ and Killeen and Fetterman (1988) have shown just some of the ways in which geometric mean bisection can be reconciled with an underlying linear time scale.

Given that distinguishing between linear and logarithmic time scales is so difficult, can any critical test be devised? Gibbon and Church (1981) argued that the time-left procedure provides such a test. The basic time-left method for pigeons is a variant of a concurrent-chain schedule, and as is normal in this type of procedure, the bird is initially faced with a choice between two concurrently available response keys (the initial links or choice links), each of which leads to a second link with the transition between the links being governed by a variable-interval schedule. When the initial link schedule terminates, the first response on the key leads to a second link, which ends with reinforcer delivery, and the other response alternative becomes unavailable. The novel feature of the time-left procedure is that the total time to reinforcement from the start of the trial (i.e., taking into account both first and second link duration) is fixed for one alternative but variable for another. One of the second links, the standard $(S)$, makes food available for the first response $S$ seconds after entering it. The other alternative, the time-left or comparison link $(C)$, makes food available after $C-t$ seconds, the "time left," where $t$ is the time of entry into the link from the start of the trial and $C$ is the total time from the start of the trial to reinforcement.

As time $(t)$ in the trial elapses, preference, as measured by relative rates of response during the concurrent initial links of the schedule, changes systematically from one alternative to the other. Early in the trial, the link leading to $S$ is generally preferred, because for short $t$ values, $C-t>S$, whereas at longer elapsed times, $C-t<S$, and a "preference for time left" develops. For example, if $S=30 \mathrm{~s}$ and $C=60 \mathrm{~s}$ (typical values from experiments with pigeons; see Gibbon \& Church, 1981), early in the trial (e.g., at $10 \mathrm{~s}$ ), $50 \mathrm{~s}$ remain until food on the alternative leading to $C$, but only 30 s remain on the alternative leading to $S$. On the other hand, when $50 \mathrm{~s}$ have elapsed, $10 \mathrm{~s}$ remain on the time-left alternative, but $S$ leads to food only in a fixed $30 \mathrm{~s}$.

How does the time-left procedure address the question of whether elapsing time is linear or logarithmic? Gibbon and Church (1981, pp. 95-96) provided an analysis in terms of changes in the point of subjective equality $\left(T_{1 / 2}\right)$ as a function of the absolute duration of the time intervals used in the experiment, where the point of subjective equality is the elapsed time in the interval for which the preference for time left is $50 \%$. The case they analyzed in detail involves experimental conditions where $S=C / 2$ (i.e., the time until food on the standard response alternative is exactly equal to half the time from the start of the trial until food on the time-left alternative). Their analysis makes a number of assumptions. First, the accumulation of subjective time as real time in the interval elapses may involve some latency, $t_{0}$; that is, some time $t_{0}$ may pass before any subjective time is accumulated, even though real time is passing. In practice, $t_{0}$ can be small, or even close to zero. Second, the choice exhibited by the animal may exhibit some multiplicative bias, $b$, which is a preference for one of the alternatives, irrespective of the time intervals involved on either of them. For example, a bird may have a certain bias toward responding on the time-left alternative, which is independent of other experimental variables.

Gibbon and Church (1981) then provided analyses of where the indifference point should be in two cases. In one of these, subjective time increases linearly with real time, and in the other it increases logarithmically. If $S=C / 2$, then for the linear case,

$$
T_{1 / 2}=(2-b) S+b t_{0},
$$

and for the logarithmic case,

$$
T_{1 / 2}=b t_{0}(C / S) \text {. }
$$

The critical factor (Gallistel, 1999; Gibbon, 1999) in the predictions of $T_{1 / 2}$ given linear or logarithmic time scaling does not lie in the roles played by $b$ and $t_{0}$, different though these are in the two equations, but in the way that $T_{1 / 2}$ depends on $S$ and $C$ when these are varied. If $S$ and $C$ vary in absolute value between conditions (recall that in the case analyzed, $S$ is always equal to $C / 2$ ), then the linear time scale predicts that $T_{1 / 2}$ should also vary. In contrast, in the logarithmic scaling case, $T_{1 / 2}$ depends only on the $C / S$ ratio, not on the absolute values of $S$ and $C$; thus, the indifference point should remain constant as $S$ and $C$ vary. Inspection of Equations 1 and 2 shows that the actual value of the indifference point for any single pair of $S / C$ values is not diagnostic of the underlying time scale, because with appropriate choice of $b$ and $t_{0}$, any value obtained experimentally could be consistent with either linear or logarithmic timing. The time-left procedure thus provides a strong test of linear versus logarithmic timing only if $S$ and $C$ are varied between conditions. Gibbon and Church (1981) tested these predictions in their Experiment 2 using a number of $S$ and $C$ values and found that $T_{1 / 2}$ changed with $S$ and $C$, supporting the contention that subjective time elapses linearly for pigeons.

Although SET was originally developed as an account of timing behavior in nonhumans, it has enjoyed considerable success in the last decade as an explanation of human timing. Allan (1998) provided a general review of some of this work, mostly that which involves what Wearden (1991a) called analogue experiments, that is, studies with humans inspired methodologically or theoretically by experiments with nonhumans (e.g., Allan \& Gibbon, 1991; Wearden, 1991b, 1992; Wearden, Denovan, Fakhri, \& Haworth, 1997; Wearden, Rogers, \& Thomas, 1997). More recently, SET has been applied to questions concerning the development of timing behavior in humans (e.g., Droit-Volet, Clément, \& Wearden, 2001; Droit-Volet \& Wearden, 2001) and has also been responsible for a revival of interest in the idea that humans might perform some timing tasks by using an "internal clock" (e.g., Wearden, Philpott, \& Win, 1999).

Just as Staddon and Higa (1999) proposed that timing in nonhuman animals was consistent with a logarithmic internal time scale, some authors have suggested that timing in humans might also be based on a logarithmic relation between subjective and real time (e.g., Brown, Preece, \& Hulme, 2000); a time-left analogue would enable the difficult question of human temporal scaling to be addressed, if a suitable technique could be devised.

Producing a time-left analogue for humans was particularly challenging, and to accomplish it, two particular problems had to 
be overcome. First, because it was impossible to imagine a realistic analogue of the time-left procedure that employs the short durations usually used in studies with humans (e.g., less than $1 \mathrm{~s}$ ), longer durations had to be substituted; therefore, some method of overcoming chronometric counting, which humans would usually use for multisecond durations, was needed. Nichelli, Alway, and Grafman (1996), Wearden, Denovan, et al. (1997), and Wearden, Rogers, et al. (1997) all successfully used a distracter task- the verbalization of temporally irregular digits presented on the computer screen-to prevent chronometric counting in humans, and that method was used here as well. Even when counting was prevented, however, a second challenge remained: how to frame the problem that the time-left procedure poses rats and pigeons in a way that is meaningful for humans.

To address this challenge, I conceived of the time-left procedure in its original form as a task in which the rat or pigeon minimizes the time taken from the start of the trial until reinforcer delivery. That is, the rat or pigeon "travels in time" from the start of the trial to reinforcement and, by taking the shortest route, obtains the reinforcer most rapidly. This idea led to a "journey minimization" analogue of the time left, shown in Figure 1.

The participant is asked to imagine taking a series of journeys by train (where each journey constitutes one trial of the task). At the station, a single train is available, and there are three possibilities for each journey. One is that the journey starts and ends on a "normal" train, which takes $C$ seconds for the trip. A second possibility is that the only train available is a "special" train. This special train completes the journey in $S$ seconds (where $S<C$ ). The special train not only is more rapid than the normal one but has the unusual characteristic that its journey time is always $S$, regardless of journey length. The third possibility is the most interesting one: The journey begins with the normal train, but after some time $t$, the special train (which always takes $S$ seconds) is potentially available and can be accepted (in which case the rest of the journey takes $S$ seconds) or rejected (in which case the normal train continues, and the remaining journey time is the "time left," $C-t$ ). In the original time-left terminology of Gibbon and Church (1981) the normal train is the comparison alternative $(C)$, and the special train, the standard alternative $(S)$.

Participants were told that the time taken to choose did not count toward journey time and were instructed to accept or reject the special train so as to minimize the total time taken for the journey. The description of the procedure was supplemented by a diagram, like that shown in Figure 1 but without any specific numerical time values. Participants were told that the choice of the special train sometimes shortened the total journey and sometimes lengthened it. No feedback could be given on the choice trials (e.g., telling participants whether their choice had minimized journey time), because such feedback would have enabled the participants to base their responses just on elapsed time in $C$, without taking any account of $S$.

\section{total $=12 \mathrm{~s} \quad$ NORMAL}

\section{"Normal" train}

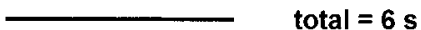

"Special" train

total $=6 \mathrm{~s}$

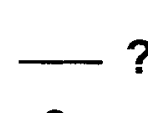

ACCEP

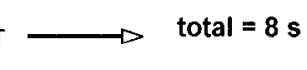

REJECT

-

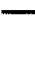

\section{?}

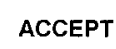

REJECT

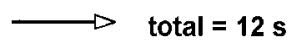

$6 \mathrm{~s}$
SPECIAL

\author{
choice at $2 \mathrm{~s}$
}

\section{choice at $6 \mathrm{~s}$}

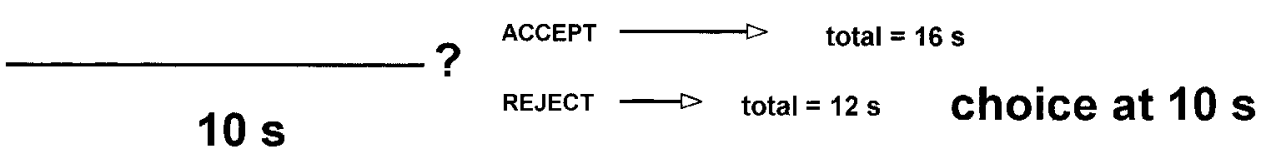

Figure 1. Schematic diagram of the time-left analogue used in Experiments 1 and 2. The condition illustrated is one in which the standard alternative $(S)$ was $6 \mathrm{~s}$ and the comparison (or time-left) alternative $(C)$ was $12 \mathrm{~s}$. Trials illustrated are a "normal" train trial and a "special" train trial (upper two lines), with the total journey time (12 s or $6 \mathrm{~s}$ ) shown. In addition, three choice trials are illustrated, where the special train is offered (indicated by ?) at $2 \mathrm{~s}, 6 \mathrm{~s}$, and $10 \mathrm{~s}$ after the start of $C$ (i.e., at entry points of $2 \mathrm{~s}, 6 \mathrm{~s}$, and $10 \mathrm{~s}$ ). The consequences of accepting or rejecting the special train (and preferring time left) on each of the different trials are shown in terms of the total journey time (in seconds). 
Some actual time values may make the procedure clearer. Suppose that the normal train journey duration is $12 \mathrm{~s}$ and the special train journey takes $6 \mathrm{~s}$, conditions that occur in Experiments 1 and 2. On some trials, the participant experiences the whole journey by the normal train (NORMAL train trial in Figure 1), and on other trials, the participant experiences the whole journey by the faster special train (SPECIAL train trial in Figure 1). On these trials there is no choice. On choice trials, the special train can be offered after the normal train journey has commenced, at various elapsed times ("entry points") into the journey, and at each of these entry points the special train can be accepted or rejected. Figure 1 shows the special train offered to participants (indicated by ?) at $2 \mathrm{~s}, 6 \mathrm{~s}$, or $10 \mathrm{~s}$ after the journey has started and also shows the consequences of accepting the special train, or preferring the time left by rejecting it. At the 2-s entry point, accepting the special train results in an 8-s total journey time, thus shortening the potential trip, whereas rejecting it results in a total of $12 \mathrm{~s}$. When the special train is offered at $6 \mathrm{~s}$ into the trial, either accepting it or rejecting it leads to the same result, a journey time of $12 \mathrm{~s}$. When the special train is offered after $10 \mathrm{~s}$, on the other hand, accepting it prolongs the trip to $16 \mathrm{~s}$, whereas rejecting it results in a shorter (12-s) trip. The optimum solution to trip minimization for any $S$ and $C$ values is easily obtained. For some $S$ value, $S$ should be accepted if $S<C-t$, where $t$ is the entry point (i.e., elapsed time in the trial), and rejected when $S>C-t$, and the indifference point $\left(T_{1 / 2}\right)$ should thus occur when $t=C-S$.

The present procedure differs from that of the normal time-left method in a number of ways. For one thing, the initial link on choice trials (i.e., the time elapsing before the choice of special and normal trains is offered) is response independent rather than being a concurrent variable-interval schedule, as in experiments with rats and pigeons. Because humans may not reliably respond like rats and pigeons on concurrent variable-interval schedules (e.g., Horne \& Lowe, 1993), allowing responding during the initial link (the measure of choice in time-left studies) added an unwanted complexity to the procedure. In the present method, only a single response (acceptance or rejection of the special train) is measured on each choice trial, so the choice index is the proportion of trials on which one or the other terminal link is entered, rather than response rates. Entry into one or the other terminal link (the special train journey or the time left on the normal train journey in our case) as a measure of choice behavior on the time-left procedure was used by Preston (1994) in his study of time left in rats, so this is not without precedent in work with nonhuman subjects, although Preston also reported the more usual measure of relative initiallink response rates.

\section{Experiment 1}

The two experiments on time left in humans in the present article address questions posed in Gibbon and Church's original time-left study (1981) and follow the lines of their Experiment 2 closely. Experiment 1 presents data from different conditions in which the special train journey length was always half that of the normal train (i.e., $S$ always equaled $C / 2$ ) and the absolute duration of the normal train journey $(C)$ was varied over values of $8 \mathrm{~s}, 12 \mathrm{~s}$, and $16 \mathrm{~s}$. As discussed above, varying the absolute values of $S$ and $C$ should systematically change the indifference point if time elapses linearly during the intervals but should not change it if time elapses logarithmically, when the $C / S$ ratio is kept constant (Equa- tion 2). Experiment 1 addresses three empirical questions. First, what do preference data from the human analogue of time left look like as $C$ is varied (with $S=C / 2$ )? Second, where is the indifference point $\left(T_{1 / 2}\right)$ located with some particular $S$ and $C$ values? Third, how does $T_{1 / 2}$ vary as $S$ and $C$ are varied between conditions?

\section{Method}

Participants. A total of 45 Manchester University first-year psychology undergraduates participating for course credit were arbitrarily allocated to three equal-sized groups. None had any knowledge of either timing or animal-learning studies at the time of the experiment

Apparatus. The experiment was conducted in a small cubicle, isolated from external lights and noise. An Opus 16X (IBM-compatible) computer with a standard color monitor controlled all experimental events. The experiment was programmed in the MEL language (Psychology Software Tools, Inc.), which ensured millisecond accuracy for timing of stimuli and responses. The computer keyboard served as the response manipulandum. A reel-to-reel tape recorder with a microphone on a stand was used to record participants' verbalizations of the distracter stimuli.

Procedure. The participants were arbitrarily allocated to three groups differing only in the values of $S$ and $C$ and the times at which the special train could be offered in choice trials, which were scaled in proportion to $C$. The groups were defined according to the value of $S$ and $C$, and $S$ was always equal to $C / 2$. This gave rise to groups $4 / 8,6 / 12$, and $8 / 16$. Consider the procedure for the $4 / 8$ group. The single experimental session (lasting about $40 \mathrm{~min}$ ) given to each participant was arranged as a series of five blocks. There were 15 trials within each block, and the order of trials varied randomly between blocks. Three trials were normal train trials, 3 were special train trials, and the remaining 9 were choice trials from which the experimental data were derived. The normal and special train trials were designed to allow the participant to experience the values of the journeys associated with each type of train on its own, in other words, to learn the values of $S$ and $C$ in isolation. The participant started the trial by pressing the space bar, then the display normal train or special train (depending on the trial) was shown in the upper part of the screen. Random digits (range of 10-99) were displayed in the center of the screen; the participant had previously been instructed to repeat these, "taking care not to miss any." The digit was displayed for $150 \mathrm{~ms}$, and the time between successive displays (offset to onset) was controlled by randomly selecting a value from two uniform distributions. One ran from 150 to $850 \mathrm{~ms}$, the other from 750 to $1,250 \mathrm{~ms}$, and both were equally likely to be used. When the time appropriate for the trial had elapsed $(8 \mathrm{~s}$ for $C$, the normal train; $4 \mathrm{~s}$ for $S$, the special train), the display journey finished was presented, and this was followed by a Press spacebar for next trial prompt.

On choice trials, the trial began in the same way as a normal train trial; that is, participants always started on the normal train. Each choice trial involved one of nine different elapsed times (entry points) before the special train was offered. For the $8 / 4$ group, these times were $0.8 \mathrm{~s}, 1.6 \mathrm{~s}, 2.4 \mathrm{~s}, \ldots 7.2 \mathrm{~s}$ from the start of the trial. When the particular time $(t)$ for the trial had elapsed, the display Accept special train? Press $Y$ or $N$ was presented, and the participant pressed the appropriate key: $Y$ to accept the special train, $N$ to reject it. After a $Y$ response, the display journey continues by special train was presented and remained on the screen; the time from that point to the journey finished display was the special train duration, $S, 4 \mathrm{~s}$ in the $4 / 8$ group. If the special train was rejected ( $N$ response), the display normal train continues was presented, and the remaining time until the journey finished prompt was the time left ( $C$ minus the time at which the choice probe had been presented). Participants were not told whether their choice had actually minimized total trial time.

As well as being exposed to the procedure described, participants initially received detailed instructions about the general method, including a diagram. It was emphasized (a) that the distracters were important and 
should be verbalized aloud, (b) that the special train always took the same length of time regardless of when it was chosen in the trial, and (c) that the aim of the participant should be to "arrive as quickly as possible." It was also emphasized that sometimes choosing the special train would make the trial shorter, whereas other times, rejecting the special train would do this, although no numerical examples citing specific times were used.

The procedure for the 6/12 and 8/16 groups was identical, except for the changes in $S$ and $C$ (to the values shown in the group identifiers) and the entry points in the choice trials at which the special train was offered. There were always nine entry points (as for the 4/8 group described above), and values were as follows: for the $6 / 12$ group, $1.2 \mathrm{~s}, 2.4 \mathrm{~s}, \ldots 10.8 \mathrm{~s}$; for the 8/16 group, $1.6 \mathrm{~s}, 3.2 \mathrm{~s}, \ldots 14.4 \mathrm{~s}$.

\section{Results}

The initial data of interest were acceptance or rejection of the special train as a function of elapsed time in the trial $(t)$, and $S$ and $C$. If the proportion of rejections is plotted against $t$, this measure is close to the "preference for time left" measure typically used in time-left procedures with rats and pigeons (e.g., Gibbon \& Church, 1981, Figure 8). Data were taken from the choice trials in the last four blocks of the session. Inspection of the preference for timeleft function for individuals revealed that the behavior of some participants was not well controlled by the procedure, probably because of its complexity. Participants were selected for inclusion according to the criterion that a participant must prefer the time left (i.e., reject the special train) more frequently for trials in which $t>$ $C / 2$ than for ones in which $t<C / 2$, thus demonstrating at least rudimentary sensitivity to the time intervals used in the experiment. This selection process rejected 17 participants in total and left 10 in the 4/8 group, 9 in the 6/12 group, and 9 in the 8/16 group.

The upper panel of Figure 2 shows the group mean preference for time left (proportion of rejections of the special train) as a function of elapsed time in the trial for the three groups. Obviously, for all three, the preference for the time-left alternative (the normal train) increased with elapsed time in the trial, from near zero at the earliest entry points to about $90 \%$ at the longest elapsed times. To analyze the data statistically, elapsed time in the interval cannot be used for the between-group comparisons, because the absolutely different $S / C$ values used mean that the groups have very few time values in common. Instead, an analysis of variance (ANOVA) of preference for time-left proportions (rejections of special train) versus entry point number $(1 \ldots 9)$ was used. Using entry point number as the independent variable rather than elapsed time in the interval effectively plots the preference for time-left functions from the different groups on the same relative scale: The scale is a relative one because the entry point values were scaled in terms of $C$ so that there were nine equally spaced values in $C$.

The ANOVA found significant effects of entry point number, $F(8,200)=74.33, p<.01$, but no effect of group, $F(1$, $25)=0.16$, nor any Group $\times$ Entry Point Number interaction, $F(16,200)=1.04$. The latter two effects show that the preference for time-left functions from the different groups (i.e., conditions with absolutely different $S$ and $C$ values) did not differ significantly in overall levels of acceptance-rejection of the time left or in overall shape, when plotted on a relative scale (i.e., against entry point number). This result shows a form of conformity to the superposition property commonly found in timed behavior in humans and nonhumans, but another test of this property is given below.
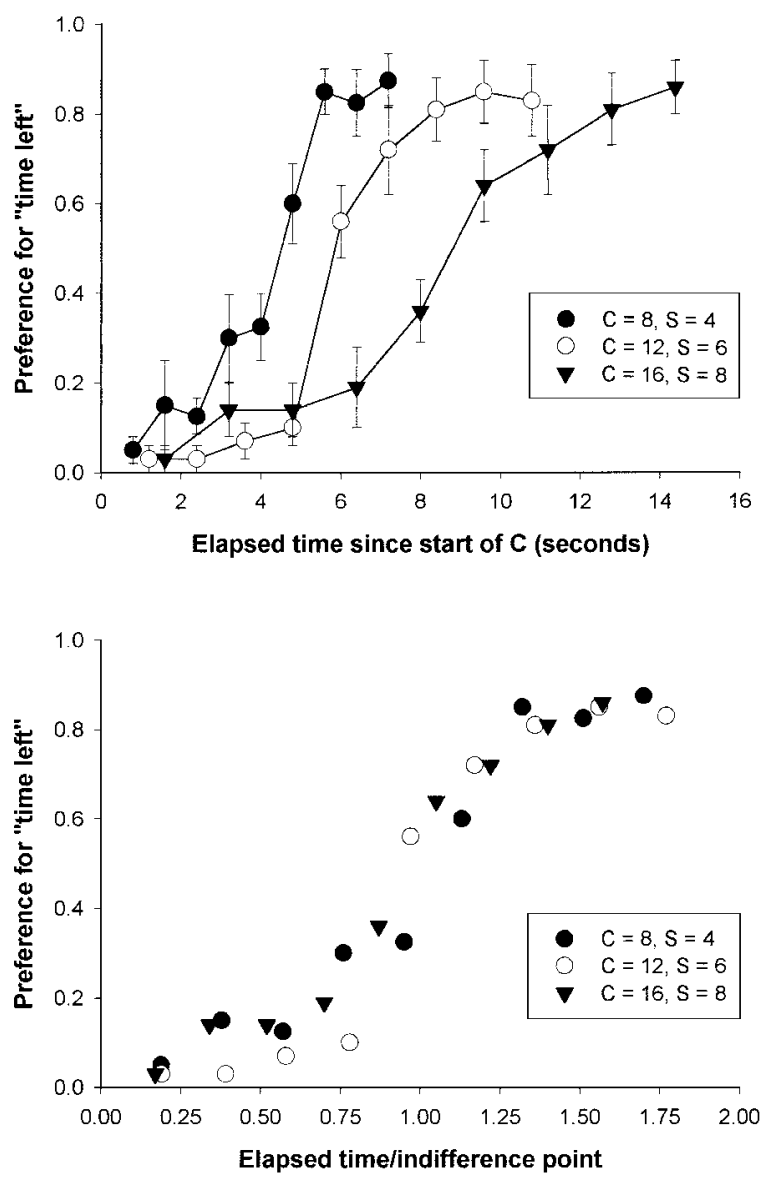

Figure 2. Data from Experiment 1. Top panel: Mean preference for the time-left alternative as a function of elapsed time in the trial. Error bars show standard error of the mean. Data are shown separately for the three groups, defined by their $S / C$ values in seconds: $4 / 8,6 / 12$, and $8 / 16$. Bottom panel: Mean preference for time left plotted against elapsed time in the trial divided by the calculated indifference point. $\mathrm{S}=$ standard alternative; $\mathrm{C}=$ comparison alternative.

Inspection of the curves relating preference to elapsed time suggests that the indifference point $\left(T_{1 / 2}\right.$, the elapsed time at which the time-left alternative was preferred on $50 \%$ of trials) changed systematically with changes in $C . T_{1 / 2}$ was calculated using a method similar to that used by Maricq, Roberts, and Church (1981) to calculate bisection points on interval bisection tasks. Linear regression was performed on the preference versus elapsed time functions for the group in question, taking data points from the region with the steepest slope (which always included values at which preference for the time-left alternative crossed from less than $50 \%$ to more than 50\%), and then the values from the regression equation were used to calculate $T_{1 / 2}$. The resulting values are shown in Table 1 , and $C-S$ is also shown. Obviously, $T_{1 / 2}$ varied systematically, and close to linearly, with changes in $C$ and $S$. In fact, $T_{1 / 2}$ values from the $4 / 8$ and $6 / 12$ groups were almost exactly equal to $C-S$, although the $T_{1 / 2}$ value from the $8 / 16$ group slightly overestimated $C-S(9.16 \mathrm{~s}$ versus $8 \mathrm{~s})$.

Regression was used to fit Equation 1 to the $T_{1 / 2}$ values shown in Table 1 , with varying $S$. With the imposed constraint that $t_{0}$ (the latency to begin the timing process) must be greater than or equal 
Table 1

Indifference Points (50\% Preference for "Time Left") in the Various Experimental Groups of Experiments 1 and 2

\begin{tabular}{ccc}
\hline Group & Indifference point (s) & $C-S(\mathrm{~s})$ \\
\hline$C=8, S=4$ & 4.23 & 4 \\
$C=12, S=6$ & 6.16 & 6 \\
$C=16, S=8$ & 9.16 & 8 \\
$C=12, S=9$ & 5.22 & 3 \\
$C=12, S=6$ & 6.64 & 6 \\
$C=12, S=3$ & 7.90 & 9 \\
\hline
\end{tabular}

Note. C is the duration (in seconds) of the "normal train" journey; $S$ is the duration of the "special train" journey. Also shown is $C-S$ (in seconds) for the different groups.

to zero, the resulting parameter values were $b=0.9$ and $t_{0}=0$, with $r^{2}=.97$. The slope of the function relating $T_{1 / 2}$ to $S$ was thus $1.1(2-b)$, as opposed to zero, the prediction consistent with logarithmic time scaling.

The lower panel of Figure 2 shows the preference versus elapsed time functions from the three groups, where preference was plotted against elapsed time divided by the calculated indifference point, $T_{1 / 2}$, for the group. This plot tests the property of superposition, a critical feature of behavior conforming to scalar timing (Gibbon, Church, \& Meck, 1984), which implies an underlying timing process with constant sensitivity as durations timed vary in absolute value. To test superposition, data from different conditions need to be plotted on the same relative scale, and the question arises of what the appropriate scale should be. The time-left procedure is a bisection-like task in the sense that preference for one response alternative over another varies as a function of time, and the measures of preference used resemble the psychophysical functions obtained from bisection tasks. The indifference point $\left(T_{1 / 2}\right)$ is a measure of central tendency that provides an appropriate unit for a relative scale plot, as it enables superposition tests of conditions in which preference versus time functions vary as a result of experimental manipulations (such as stimulus spacing effects on bisection with humans; see Wearden \& Ferrara, 1995). Scaling the preference functions by $T_{1 / 2}$ is thus a more appropriate test of the superposition property than scaling entry point values by $C$ (see also Allan \& Gibbon, 1991, for discussion of tests of superimposition in bisection). Inspection of data from the three groups suggests that superimposition held reasonably well, in spite of the fact that the task was a complicated one.

\section{Discussion}

Experiment 1 mirrored some conditions from Gibbon and Church's (1981) Experiment 2, where pigeons received a series of time-left schedules, in most of which $S$ was equal to $C / 2$. The time values used in their study with pigeons, however, were always longer than those used here, sometimes substantially so, with $S / C$ values ranging from $15 / 30 \mathrm{~s}$ to $90 / 180 \mathrm{~s}$. In addition, pigeons in their study were exposed to a number of conditions rather than just one, as the present participants were. Another difference was that preference was measured in terms of relative response rates during the initial link of the concurrent chains procedure rather than in terms of entries into the second link, as here (but see Preston, 1994). In spite of these procedural differences, the general details of the present results were strikingly similar to those obtained from pigeons.

A first point of similarity was in the preference functions themselves. Comparing the upper panel of the present Figure 2 with Figure 8 from Gibbon and Church (1981) reveals very similar performance. In both species, preference for the time-left alternative increased with elapsed time in the trial and, as $C$ and $S$ lengthened between conditions, the preference function shifted systematically rightward. Second, $T_{1 / 2}$ values in Gibbon and Church's study (their Figure 9) systematically varied with $S$ and $C$, although they deviated much more from $C-S$ than did those derived from the present data. Fitting Equation 1 to their data resulted in a $b$ of 1.26 (i.e., a slope of 0.74 ) and a $t_{0}$ of $0.49 \mathrm{~s}$, a negligibly small value compared with the durations of $C$ and $S$ used. The $b$ value obtained in their study indicated a substantial bias toward the time-left alternative, whereas data from the present Experiment 1 were slightly biased in the opposite direction. Irrespective of these differences in bias, the change in $T_{1 / 2}$ with changes in $S$ and $C$ clearly supported linear growth of subjective time in both people and pigeons.

As a final point of comparison, although Gibbon and Church (1981) did not provide superposition graphs, Gibbon, Church, Fairhurst, and Kacelnik (1988) presented superposition data from a number of time-left conditions with pigeons in which $S=C / 2$ (with $S$ ranging from $7.5 \mathrm{~s}$ to $60 \mathrm{~s}$ ) in their Figure 5. When preference functions were plotted against elapsed time divided by $T_{1 / 2}$, the functions from pigeons superposed nearly perfectly. Although the quality of superposition in pigeons was superior to that exhibited by participants in Experiment 1 (lower panel of the present Figure 2), it is clear that superposition generally held in both species.

Although it is clear that the time-left analogue for humans produced generally orderly data, the procedure was technically problematic in some respects, particularly compared with the ease of obtaining extremely orderly data from humans in other analogues of animal timing experiments, such as bisection (Wearden \& Ferrara, 1995, 1996; Wearden, Rogers, et al., 1997) and temporal generalization (Wearden, 1992; Wearden, Denovan, et al., 1997). In time left, not only did some participants apparently have difficulty understanding the complex instructions and procedure, but even those who appeared to do so spontaneously reported that they found the task very demanding and that it was particularly difficult to maintain attention to the task during the experimental session. Even by the standards of other timing experiments with humans, the experimental trials in the time-left analogue were very sparse in terms of stimuli presented and responses made. There were no stimuli to be timed, as the only stimuli provided were the distracter stimuli and the displays indicating the train in force. No feedback could be given, as noted above, and on six trials in each block there was not even the single response that was required on the other nine trials. Furthermore, trials in some conditions (e.g., the 8/16 group) could last more than $16 \mathrm{~s}$, considerably longer than those used in most timing studies with humans inspired by animal experiments.

It was difficult, however, to devise a method of making the task more engaging for participants. One possibility was to have some kind of within-trial event that marked the passage of time, for example, a change of screen background representing changing scenery in the imaginary journey or even the movement of a small animated train across the screen. However, this would obviously 
have provided participants with some nontemporal cue that they could use to make their acceptance or rejection decisions, and so it was precluded. In a pilot study I attempted to increase the orderliness of the data by exposing participants to more than one session, a common strategy in psychophysical timing tasks (e.g., Allan \& Gibbon, 1991). However, this was unsuccessful, as participants reported that it was easier to concentrate on the task in the first session when the procedure was novel, rather than in later ones.

\section{Experiment 2}

Experiment 2 addresses another question touched on by Gibbon and Church (1981) in their Experiment 2, that of what behavior looks like on the time-left procedure when $S$ is not equal to $C / 2$. In the present Experiment 2, data were collected when $S$ was $C / 4$, $C / 2$, and $3 C / 4$. In experiments with rats and pigeons, the overwhelming majority of data come from conditions where $S=C / 2$, but some other values have been explored. Gibbon and Church reported a small amount of data from conditions with $S=C$ (with both values $60 \mathrm{~s}$ ) and $S=C / 4$, with $C=60 \mathrm{~s}$, and Preston (1994) presented data from rats with $S=C / 3$, when $C$ was $90 \mathrm{~s}$. What these studies found, and what the implications of their results are, are discussed later.

In Experiment 2, three different groups of participants received time-left conditions with $S / C$ pairs of $3 / 12 \mathrm{~s}, 6 / 12 \mathrm{~s}$, and $9 / 12 \mathrm{~s}$, with entry points at $1,2,3, \ldots 11 \mathrm{~s}$ into the trial.

\section{Method}

Participants. A total of 45 Manchester University first-year undergraduates, participating for course credit, were arbitrarily allocated to three equal-sized groups.

Apparatus. The apparatus was as described in Experiment 1.

Procedure. The procedure was identical in almost all respects to that used for the 4/8 group of Experiment 1 except that the $S / C$ values (in seconds) for the various conditions were $3 / 12,6 / 12$, and $9 / 12$. The entry points were $1 \mathrm{~s}, 2 \mathrm{~s}, 3 \mathrm{~s}, \ldots 11 \mathrm{~s}$ into the trial. Each block consisted on 3 normal train trials, 3 special train trials, and 11 choice trials, presented in a random order within five blocks. All other details were as in Experiment 1.

\section{Results}

As in Experiment 1 the behavior of some participants was not well controlled by the procedure, so the same criterion as in the previous experiment was used to discard some data. This selection process left 10 participants in the 9/12 group, 10 in the 6/12 group, and 14 in the 3/12 group. The upper panel of Figure 3 shows preference for the time-left alternative as a function of elapsed time in the trial, with data coming from the last four blocks of the experiment.

In Experiment 2, the entry points were the same elapsed times in the trial for all groups, so an analysis in terms of absolute elapsed time and entry point number was identical. An ANOVA of the preference for time left against elapsed time in the trial found significant main effects of elapsed time, $F(10,310)=104.4, p<$ .01 , and group, $F(1,31)=20.56, p<.01$, and a significant Group $\times$ Elapsed Time interaction, $F(20,310)=2.13, p<.01$. The first of these results confirms the effect obvious in Figure 3 that elapsed time in the trial influenced preference for time left, the
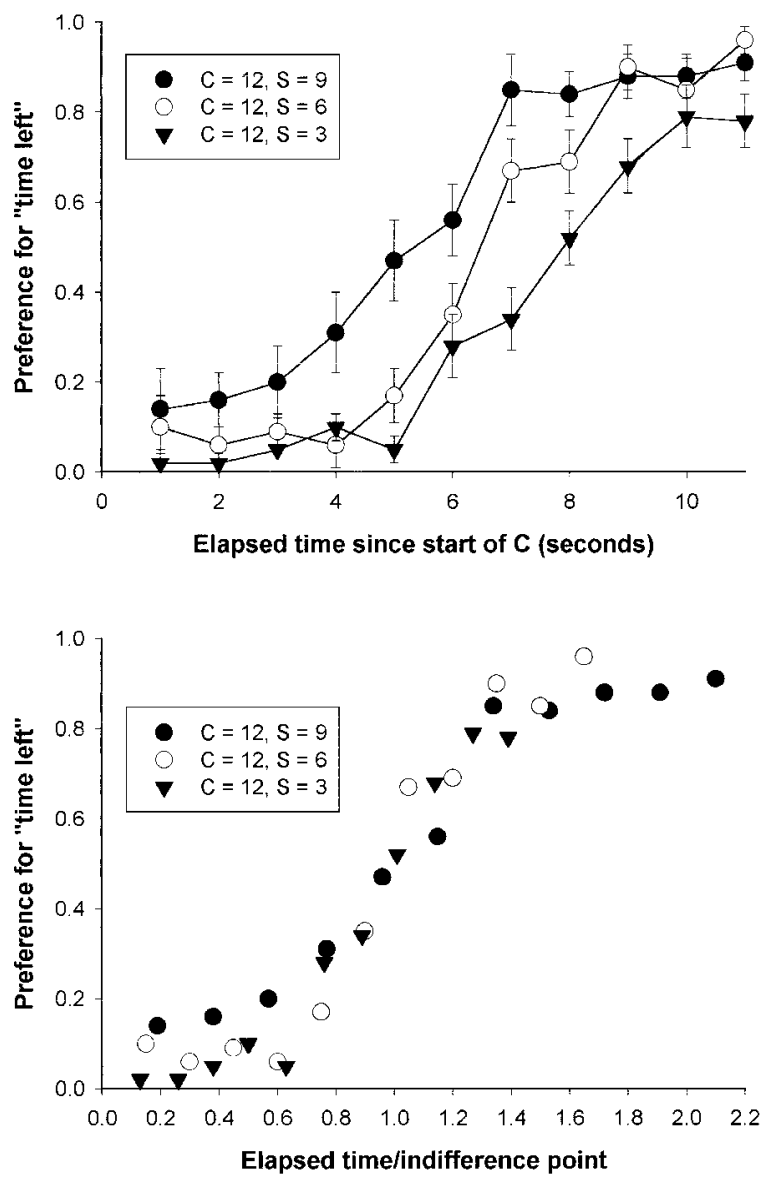

Figure 3. Data from Experiment 2. Top panel: Mean preference for the time-left alternative as a function of elapsed time in the trial. Error bars show standard error of the mean. Data are shown separately for the three groups, defined by their $S / C$ values in seconds: 9/12, 6/12, and 3/12. Bottom panel: Mean preference for time left plotted against elapsed time in the trial divided by the calculated indifference point. $\mathrm{S}=$ standard alternative; $\mathrm{C}=$ comparison alternative.

second confirms that the different conditions produced different degrees of preference for time left overall (e.g., time left was preferred more overall in the 9/12 group than in the 3/12 one), and the third that the time-left preference varied as a function of elapsed time in a way that was different between conditions (i.e., the participants were sensitive to the relation between $S$ and $C$ ).

$T_{1 / 2}$ was determined in the same manner as in Experiment 1, and the resulting values are shown in Table 1 . As $S$ (and thus $C-S$ ) varied, $T_{1 / 2}$ changed systematically, exceeding $C-S$ when $S=9 \mathrm{~s}$, being close to it when $S=6 \mathrm{~s}$, and undershooting $C-S$ when $S=$ $3 \mathrm{~s}$. In other words, participants tended to reject the special train too early in the trial when $S=3 \mathrm{~s}$ and to reject it too late when $S=$ $9 \mathrm{~s}$, whereas when $S=6 \mathrm{~s}, T_{1 / 2}$ was close to $C-S$.

The lower panel of Figure 3 shows the preference for time left plotted against elapsed time functions, when elapsed time was expressed as a fraction of the $T_{1 / 2}$ value calculated for the condition in force (i.e., a plot that expresses the quality of superposition found in the data). As in Experiment 1, superposition was generally good. 


\section{Discussion}

In Experiment 2, preference for time left increased systematically with increasing elapsed time in the interval. When $S=9$ s, participants on average did not reject $S$ early enough (i.e., they prolonged the journey unnecessarily by accepting the special train), whereas when $S=3 \mathrm{~s}$, they prolonged the journey unnecessarily by rejecting $S$ too early in the trial, compared with the "ideal" performance (discussed above) of rejecting $S$ when $\mathrm{S}>C$ $-t$ and accepting it when $S<C-t$.

Data from rats and pigeons on simple time-left tasks when $S$ is not equal to $C / 2$ are scarce (although such conditions have been used in more complex forms of the task by Gibbon, 1986, and Brunner, Gibbon, \& Fairhurst, 1994). Gibbon and Church (1981) reported only two conditions of this sort. In one of these, $S / C$ values were $15 / 60 \mathrm{~s}$, and in the other the values were both $60 \mathrm{~s}$ (i.e., $S$ was the same length as $C$ ). Inspection of the data in these two conditions (Gibbon \& Church, 1981, Figure 11) reveals a pattern of results reminiscent of those obtained in the present Experiment 2: When $S$ was $15 \mathrm{~s}, T_{1 / 2}$ was about $35 \mathrm{~s}$ (lower than $C-S, 45$ s), so $S$ was rejected too early, whereas when $S$ and $C$ both equaled $60 \mathrm{~s}, T_{1 / 2}$ was about $8 \mathrm{~s}$, rather than zero, which is $C$ $-S$, so in this case the pigeons rejected $S$ too late. Preston (1994, Experiment 1) reported data from rats with the $S / C$ pair $30 / 90 \mathrm{~s}$ and measured preference for time left in terms of both relative response rates on the response alternatives leading to $S$ and $C$ and entries to $S$ and $C$, a measure much more closely resembling the choice measure used in the present article. Across different rats, $T_{1 / 2}$, when measured by response rates, undershot $C-S(60 \mathrm{~s})$, in 3 rats (values of $46.7 \mathrm{~s}, 41.5 \mathrm{~s}$, and $51.0 \mathrm{~s}$ ), but was closer to it (56.1 s) in another rat. $T_{1 / 2}$ values derived from entries were not given numerically by Preston, but from his Figure 3 it is clear that these were usually closer to $60 \mathrm{~s}$ than the value derived from response measures, and in the case of 3 out of 4 rats, within a few seconds of $60 \mathrm{~s}$.

Overall, therefore, what data exist from rats and pigeons generally support the picture obtained from the present Experiment 2. Although there has been little systematic manipulation of the effects of the $S / C$ ratio on time left in studies with nonhumans, the few existing data (from Gibbon \& Church, 1981, Figure 11) do suggest that when $S<C / 2$, pigeons rejected $S$ too early, and when $S>C / 2$ they rejected it too late, the same pattern of results as shown by participants in the present Experiment 2 .

\section{General Discussion}

The experiments above show that the analogue of the time-left procedure can produce not only orderly data but also results that bear a clear resemblance to data previously obtained from rats and pigeons and that can therefore be used to address the same theoretical issues. By far the most important of these is that of whether subjective time appreciates linearly or logarithmically in this paradigm. By demonstrating a clear change in $T_{1 / 2}$ when $C$ is varied in conditions with $S=C / 2$ (the present Experiment 1), results presented here clearly support Gibbon and Church's (1981) contention that subjective time appreciates as a linear function of clock time, rather than logarithmically. As mentioned above, several recent theoretical models that apply directly to timing behavior (Staddon \& Higa, 1999) or that use underlying timing mechanisms to predict other sorts of behavior (Brown et al., 2000) have assumed an underlying logarithmic time scale, which is contrary to the clear implications of the results of the present work.

Another subsidiary result of the present work is the suggestion (supported by some data from Gibbon \& Church's, 1981, time-left study with pigeons) that preference for or against time-left alternatives might (a) be biased and (b) exhibit changes that depend on the relation between $C$ and $S$.

Both Gibbon and Church (1981) and Preston (1994) reported a strong preference for the time-left link in almost all data from their pigeons and rats (particularly when measured in terms of relative response rates; see Preston, 1994), although this bias was much less marked in the present Experiment 1, where the indifference point was close to $C-S$. Why does this preference occur in nonhumans?

One possibility is that it is a reflection of rats' and pigeons' well-known preference for variable, as opposed to fixed, delays of reinforcement (Killeen, 1968; see also Gibbon, Church, Fairhurst, et al., 1988, for review and discussion). When the $S$ link is entered, the reinforcer becomes available after a fixed $S$ seconds, whereas the length of time to reinforcement on the time-left link $(C-t$ seconds) depends on when the link was entered and is thus variable. So, even in the case where $S$ is on average equal to $C / 2$, the fact that the link associated with $C$ provides reinforcers after variable times may lead to a preference for $C$. Gibbon, Church, Fairhurst, et al. (1988) tested this idea, in their Experiment 1, using a time-left procedure in which the standard $S$ was either a fixed $30 \mathrm{~s}$ or a variable period averaging $30 \mathrm{~s}$ and in which preference for $S$ was markedly increased in the latter case. In their treatment of the standard time-left procedure, Gibbon and Church (1981) model the preference for the time-left alternative as a bias, that is, as a constant preference for that alternative regardless of other schedule factors, a usage reminiscent of the use of the term bias in analyses of matching, derived initially from Baum (1974).

However, this usage is much less consistent with Gibbon and Church's (1981) results when $S$ was not equal to $C / 2$. With $S=$ $C / 4$, the bias toward the time left was even greater than that shown with $S=C / 2$, whereas with $S=C$, the pigeons were biased toward $S$ and thus away from the time-left link. This latter case is not easy to reconcile with the "preference for variability" argument taken above from Gibbon, Church, Fairhurst, et al. (1988), because not only does $S$ provide a fixed time to the reinforcement compared with the variable time on the time-left alternative, but once the trial has started, the time to reinforcement $(C-t)$ on the time-left alternative is actually shorter than the time to reinforcement on $S$.

In the present Experiment 1, the choice responses of our participants appeared to be almost unbiased in Gibbon and Church's (1981) sense. In Experiment 2, in contrast, the balance of preference toward and away from $C$ appeared to depend on the $S / C$ ratio (i.e., $S$ was rejected too early with $S=C / 4$ and too late with $S=$ $3 C / 4$ ), and this change of preference seems also inconsistent with the term bias as Gibbon and Church used it. However, given the paucity of data from conditions in which the $S / C$ ratio has been varied in studies with nonhumans, it is unclear whether this type of effect arises from some peculiarity of the present procedure or the species used or whether it is some general property of responding on the time-left procedure that deserves further experimental and theoretical attention, although the material in Gibbon and Church's Figure 11 strongly suggests the latter.

The present analogue of the time-left procedure adds to a growing body of work on human timing influenced by studies of 
animal timing (some of which is reviewed by Allan, 1998), work that has recently been extended to include studies of timing behavior in humans as young as 3 years of age (Droit-Volet et al., 2001; Droit-Volet \& Wearden, 2001), in which properties exhibited in animal timing, such as superposition, appear to hold. There can be few areas of animal behavior processes that have had such a strong methodological and theoretical influence on studies with humans as recent studies of animal timing.

\section{References}

Allan, L. G. (1998). The influence of the scalar timing model on human timing research. Behavioural Processes, 44, 101-117.

Allan, L. G., \& Gibbon, J. (1991). Human bisection at the geometric mean. Learning and Motivation, 22, 39-58.

Baum, W. M. (1974). On two types of deviation from the matching law: Bias and undermatching. Journal of the Experimental Analysis of Behavior, 22, 231-242.

Brown, G. D. A., Preece, T., \& Hulme, C. (2000). Oscillator-based memory for serial order. Psychological Review, 107, 127-181.

Brunner, D., Gibbon, J., \& Fairhurst, S. (1994). Choice between fixed and variable delays with different reward amounts. Journal of Experimental Psychology: Animal Behavior Processes, 20, 331-346.

Church, R. M., \& Deluty, M. Z. (1977). Bisection of temporal intervals. Journal of Experimental Psychology: Animal Behavior Processes, 3, 216-228.

Droit-Volet, S., Clément, A., \& Wearden, J. H. (2001). Temporal generalization in 3- to 8-year-old children. Journal of Experimental Child Psychology, 80, 271-288.

Droit-Volet, S., \& Wearden, J. H. (2001). Temporal bisection in children. Journal of Experimental Child Psychology, 80, 142-159.

Eisler, H. (1975). Subjective duration and psychophysics. Psychological Review, 82, 429-450.

Eisler, H. (1976). Experiments on subjective duration 1868-1975: A collection of power function exponents. Psychological Bulletin, 83, $1154-1171$

Gallistel, C. R. (1999). Can a decay process explain the timing of conditioned responses? Journal of the Experimental Analysis of Behavior, 71, 264-271.

Gibbon, J. (1981). Two kinds of ambiguity in the study of psychological time. In M. A. Commons \& J. A. Nevin (Eds.), Quantitative analyses of behavior: Vol. 1. Discriminative properties of reinforcement schedules (pp. 157-189). Cambridge, MA: Ballinger.

Gibbon, J. (1986). The structure of subjective time: How time flies. In G. H. Bower (Ed.), The psychology of learning and motivation (Vol. 20, pp. 105-135). Orlando, FL: Academic Press.

Gibbon, J. (1999). Multiple time scales is well named. Journal of the Experimental Analysis of Behavior, 71, 272-275.

Gibbon, J., \& Church, R. M. (1981). Time left: Linear versus logarithmic subjective time. Journal of Experimental Psychology: Animal Behavior Processes, 7, 87-108.

Gibbon, J., Church, R. M., Fairhurst, S., \& Kacelnik, A. (1988). Scalar expectancy theory and the choice between delayed rewards. Psychological Review, 95, 102-114.

Gibbon, J., Church, R. M., \& Meck, W. (1984). Scalar timing in memory. In J. Gibbon \& L. Allan (Eds.), Annals of the New York Academy of Sciences: Timing and time perception (Vol. 423, pp. 52-77). New York: New York Academy of Sciences.

Horne, P. J., \& Lowe, C. F. (1993). Determinants of human performance on concurrent schedules. Journal of the Experimental Analysis of Behavior, 59, 29-60.

Killeen, P. (1968). On the measurement of reinforcement frequency in the study of preference. Journal of the Experimental Analysis of Behavior, 11, 263-269.

Killeen, P., \& Fetterman, J. G. (1988). A behavioral theory of timing. Psychological Review, 95, 274-295.

Lowe, C. F., Harzem, P., \& Spencer, P. T. (1979). Temporal control of behavior and the power law. Journal of the Experimental Analysis of Behavior, 31, 333-343.

Maricq, A. V., Roberts, S., \& Church, R. M. (1981). Metamphetamine and time estimation. Journal of Experimental Psychology: Animal Behavior Processes, 7, 18-30.

Marks, L. E. (1974). Sensory processes: The new psychophysics. London: Academic Press.

Nichelli, P., Alway, D., \& Grafman, J. (1996). Perceptual timing in cerebellar degeneration. Neuropsychologia, 34, 863-871.

Platt, J. R. (1979). Temporal differentiation and the psychophysics of time. In M. D. Zeiler \& P. Harzem (Eds.), Reinforcement and the organisation of behavior (pp. 1-29). Chichester, England: Wiley.

Preston, R. A. (1994). Choice in the time-left procedure and in concurrent chains with a time-left terminal link. Journal of the Experimental Analysis of Behavior, 61, 349-373.

Staddon, J. E. R., \& Higa, J. J. (1999). Time and memory: Towards a pacemaker-free theory of interval timing. Journal of the Experimental Analysis of Behavior, 71, 215-251.

Stevens, S. S. (1957). On the psychophysical law. Psychological Review, 64, 153-181.

Wearden, J. H. (1985). The power law and Weber's law in fixed-interval postreinforcement pausing: A scalar timing model. Quarterly Journal of Experimental Psychology, 37B, 191-211.

Wearden, J. H. (1991a). Do humans possess an internal clock with scalar timing properties? Learning and Motivation, 22, 59-83.

Wearden, J. H. (1991b). Human performance on an analogue of an interval bisection task. Quarterly Journal of Experimental Psychology, 43B, $59-81$.

Wearden, J. H. (1992). Temporal generalization in humans. Journal of Experimental Psychology: Animal Behavior Processes, 18, 134-144.

Wearden, J. H. (1999). "Beyond the fields we know. . .": Exploring and developing scalar timing theory. Behavioural Processes, 45, 3-21.

Wearden, J. H., Denovan, L., Fakhri, M., \& Haworth, R. (1997). Scalar timing in temporal generalization in humans with longer stimulus durations. Journal of Experimental Psychology: Animal Behavior Processes, $23,502-511$.

Wearden, J. H., \& Ferrara, A. (1995). Stimulus spacing effects in temporal bisection by humans. Quarterly Journal of Experimental Psychology, 48B, 289-310.

Wearden, J. H., \& Ferrara, A. (1996). Stimulus range effects in temporal bisection by humans. Quarterly Journal of Experimental Psychology, 49B, 24-44.

Wearden, J. H., Philpott, K., \& Win, T. (1999). Speeding up and (. . relatively...) slowing down an internal clock in humans. Behavioural Processes, 46, 63-73.

Wearden, J. H., Rogers, P., \& Thomas, R. (1997). Temporal bisection in humans with longer stimulus durations. Quarterly Journal of Experimental Psychology, 50B, 79-94.

Received April 2, 2001

Revision received December 10, 2001

Accepted December 11, 2001 